Traditional Medicine and Modern Medicine

Vol. 2, No. 2 (2019) 43-47

(C) Institutes of Integrative Medicine, Fudan University and

World Century Publishing Corporation

DOI: $10.1142 /$ S2575900019200015

\title{
Interpretation of the changes in 2018 National Essential Medicine List (NEML) from the specialists
}

\author{
Chuyu $\mathrm{Li}^{\dagger}$, Xinyao Pan ${ }^{\dagger}$, Wingting Leung, Zengshu Huang, \\ Jing Zhou and Ling Wang* \\ Laboratory for Reproductive Immunology \\ Hospital \& Institute of Obstetrics and Gynecology \\ Shanghai Medical College, Fudan University \\ Shanghai, P. R. China \\ The Academy of Integrative Medicine of Fudan University \\ Shanghai, P. R. China \\ Shanghai Key Laboratory of Female Reproductive \\ Endocrine-related Diseases, Shanghai, P. R. China \\ *Dr.wangling@fudan.edu.cn
}

Received 21 May 2019; Accepted 29 May 2019; Published 4 July 2019

\begin{abstract}
On 25 October 2018, National Essential Medicine List (NEML) of China was formally published by National Health Commission and it has been executed since 1 November. The new NEML, which includes 685 drugs with more than 1110 kinds of dosage forms and more than 1810 kinds of specifications, further standardizes dosage forms and specifications of drugs. The new catalog not only increases the number of categories, but also optimizes the structure of the drug list. It highlights the need for basic drugs in aspects of common diseases, chronic diseases, serious diseases and public health. Besides, the normalization of dosage forms and specifications, and the persistence of emphasizing the combination of Western and Chinese medicine are both the characteristics of it. This news report is composed of several parts including brief introduction of NEML, the difference between NEML and National Directory of Health Insurance (NDHI) which is likely to be confused with NEML, the dissection of the change of medicine varieties, relevant policies and potential issues of NEML. We intend to give a comprehensive interpretation of NEML from different perspectives.
\end{abstract}

Keywords: National Essential Medicine List (NEML); medicine; China.

\section{Introduction}

According to World Health Organization (WHO), Essential Medicines are defined as "most important, basic, indispensable and necessary drugs for the health and needs of the population". ${ }^{1}$ They are supposed to be obtainable at any time. The amounts, quality, appropriate dosage forms and affordable ability of the public should be guaranteed. ${ }^{2}$ The initial purpose of listing essential medicine was providing basic medicine at an affordable price under the limitation of medical resources, especially for underdeveloped countries or regions. $^{3}$ Inspired by National Essential Medicine List (NEML) policy of WHO and on the basis of the empirical evidence from the domestic doctors, China established its own NEML policy in 2009. This policy is aimed at realizing the $100 \%$ availability of essential medicines in primary hospitals in both urban and rural areas. ${ }^{4}$ 


\section{The Distinction Between NEML and NDHI}

\section{Concept and goals}

The aim of NEML is to improve the quality of life of all the residents and to ensure the availability of medicine to pharmacists, medical staffs and all the residents. It is designed to depress the surging drug prices, to prompt the reform of medical insurance system, to instruct medical stuff to choose proper drug varieties and to ensure the market supplement of essential medicine by means of guiding the productive direction of drug producing enterprises. On the other hand, the National Directory of Health Insurance (NDHI) also aims to control the range of the cost covered by health insurance, to ensure the fundamental need of people participating in the insurance, to alleviate the expenditure burden of health insurance foundation and to keep the balance of income and expenditure of insurance foundation. Although they are both made to depress the fast-rising prices of drugs and help residents to use medicine properly, NEML aims at all the residents, whereas NDHI only fits for residents covered by medical insurance. Besides, NEML controls cost of drugs, by comparison, NDHI limits cost of insurance.

\section{The inclusion criteria of NEML}

The inclusion criteria of NEML and NDHI are almost the same: necessity, safety and affordability. However, NEML mainly considers the rationality and safety of clinical use of drugs as well as the basic drug use level of the whole society. It emphasizes clinical first choice and requires that the drugs must have representativeness and are obtainable basic health organizations, in order to meet with the health requirement of all the residents. NDHI not only takes the safety and efficacy into consideration, but at the same time, it is also based on the tolerating ability of health insurance and it puts more emphasis on the costs of drugs.

\section{Executing efficacy}

NEML instructs doctors to use drugs properly by means of educating the society and training the medical stuff, whereas $\mathrm{NDHI}$ is executed when social insurance agencies pays for the cost. $^{5}$

\section{The Dissection of the Change of Medicine Varieties}

The adjustment of the NEML is an optimized decision based on the basic national conditions of China, including the economic and social development level, the medical security level, the change of disease spectrum and the basic medical and health needs. It takes the development of both science and technology into account. ${ }^{4,6}$

\section{Cancer}

In order to ensure that cancer patients have access to affordable medicines, China has also introduced new policies. It is reported that 12 kinds of anticancer drugs have been added to the list of essential drugs this time, which increases the number of kinds of anticancer drugs to 38 . The new edition includes some new drugs such as small-molecule targeted drugs, which has brought good news to cancer patients. Trastuzumab for injection, which was newly included in the coverage of medicare in 2017, is one of the small-molecule targeting antitumor drugs newly introduced into NEML. ${ }^{7,8}$ At present, breast cancer has become the malignant tumor with the highest incidence rate among women in the world. About 1.2 million women around the world suffer from breast cancer every year. ${ }^{9}$ Due to factors such as long research period, high expenditure, high tariff and other reasons, the price of imported targeting drugs is higher than the conventional chemotherapy drugs. When patients with cancer select targeted therapy, they also begin to bear a heavy economic burden at the same time. To some extent, this has limited the application of targeted drugs. Since tumor targeting drugs were included in the scope of medical insurance payment, the price is $40 \%$ lower than the original cost, and patients only need to pay part of the remnant, which will greatly reduce the burden of medical expenses for cancer patients in China. ${ }^{10}$

It is reported that since the beginning of 2018, in addition to adding new anticancer drugs to the list of basic drugs, China has successively introduced corresponding policies. The policies include zero tariff on anticancer drugs, centralized government procurement through negotiation, inclusion of anticancer drugs in the list of medical insurance reimbursement and acceleration of the import and marketing of innovative drugs. The release of these policies has brought many benefits to cancer patients.

\section{Traditional Chinese medicine}

Traditional Chinese medicine (TCM) is the treasure and essence of 5000 years' traditional Chinese culture. It has not only made indelible contributions to the prosperity of the Chinese nation, but also had a positive impact on human health and world civilization. In recent years, the appeal to support the development of TCM industry is also rising. It is reported that Measures for the Administration of the NEML (No. 52 [2015] issued by the national health administration of the People's Republic of China) clearly stipulate that both Chinese and Western medicines should be emphasized during 
the selection of national essential drugs. The adjustment principle set out in the work plan for the adjustment of the 2018 NEML also clearly supports the development of TCM. For example, from the perspective of the number of newly added children's medicines, the proportion of Chinese patent medicines is close to $64 \% .{ }^{11}$ Nowadays, the development of TCM industry is entering into the fast track. With the introduction of Western medicine and the export of TCM, the Chinese and Western medicine can be combined together and complement each other. In recent years, as China continues to promote the development of TCM industry, the industry will prosper soon.

\section{Obstetrics and gynecology}

Four kinds of drugs for Obstetric and gynecological use have been included in NEML in the new edition. They are Carboprost Methylate, Bu Xue Yi Mu Wan, Dingkundan Capsule and Zishen Yutai Pill. The vaginal administration of Carboprost methylate can induce the contraction of uterine smooth muscle and expansion of cervix. It can do harm to fetus and placenta, which may lead to the decrease of blood progesterone level and synthesis of endogenous prostaglandin. Then high frequency and high amplitude contraction of uterus similar to normal labor process can be triggered by the changes of the hormones, achieving abortion or labor induction. The main ingredients of $\mathrm{Bu}$ Xue Yi Mu Wan are angelica, astragalus, donkey-hide gelatin, motherwort and tangerine peel. It has the function of invigorating $Q i$ and Blood, removing stasis and creating New. It is used for postpartum abdominal pain caused by deficiency of $Q i$ and Blood and blood stasis syndrome. Dingkundan Capsule nourishes $Q i$ and Blood, regulating menstruation and relieving depression. It is used for abdominal pain caused by deficiency of $Q i$ and blood, stagnation of $Q i$ and blood stasis. Zishen Yutai Pill can tonify kidney and spleen, invigorating $Q i$ and cultivating Yuan, nourishing blood and placating fetus, strengthening the body. It is used to prevent and treat recurrent abortion and threatened abortion. Thanks to the improving concern for health and opening of the two-child policy, obstetrics and gynecology diseases attract more attention in many fields of our society. The inclusion of these drugs has shown special concern for women from medical field and aims to improve the quality of life of women.

\section{Pediatrics}

The demand of specific crowds such as children did not get much attention in the previous NEML. In this revision, the need of this special group has been taken full consideration. In addition to adding dosage forms and specifications suitable for children under the original generic name, the new catalog also added new generic names of special medicines. For example, the new NEML included the lung surfactants for the treatment of neonatal respiratory distress syndrome, Pegaspargase for the treatment of acute lymphoblastic leukemia in children, etc.

\section{Antimicrobial drugs}

In the past, interferon was the priority for the treatment of hepatitis $\mathrm{C}$ in our country, but toxic reaction was obvious and cure rate was low. Many patients with hepatitis $\mathrm{C}$ also used generic or counterfeit drugs from countries such as India in which treating effect could not be guaranteed. Sofosbuvir and Vipantavil tablets are new drugs for the treatment of hepatitis C, which are completely for oral administration. ${ }^{6,12}$ They were approved for marketing in May 2018. As the world's most advanced drugs for treating hepatitis C, Sofosbuvir and Vipantavil tablets could theoretically cure about $80 \%$ of hepatitis $\mathrm{C}$ patients in China. The inclusion of the two medicine has a clear clinical need and helps speed up the accessibility of the drug through multiple policy interactions.

\section{Medical Insurance Linkage Promotes Drug Price Reduction}

At an executive meeting of the state council on 30 August 2018, the state council proposed to promote centralized procurement by public medical institutions in order to lower drug prices. It will establish a mechanism for linking basic drugs with basic medical insurance and ensuring its sustainability. It will give priority to the inclusion of eligible therapeutic drugs from NEML into the list of medical insurance, so more expenditure of patients will be covered by medical insurance. It will encourage all localities to give priority to the use of basic drugs in the management of chronic diseases such as hypertension, diabetes mellitus and severe mental disorders, and reduce drug costs for patients while ensuring their effectiveness. While exerting the guiding role of the catalog of essential drugs, we should allow all localities to take the reality into consideration and keep the balance of expenses and receipts to focus on ensuring basic medical insurance and to effectively reduce the burden on patients. $^{13}$

According to the previous draft, the use of essential drugs will be linked to the allotment of subsidy funds for the reform of public hospitals and the implement of basic essential medicine system. At the same time, the government will deepen reform of the way in which medical insurance is paid, and give priority to setting medical insurance payment standards for basic drugs. For the essential drugs used in clinical diagnosis and treatment, after the relevant procedures of the medical insurance department, the drugs are all 
included in the reimbursement list, and the reimbursement proportion is significantly higher than that of non-essential drugs.

What is worth mentioning is that China will also provide some essential drugs for free to patients with hypertension, diabetes and serious mental disorders by means of full financial guarantee or full reimbursement of medical insurance. Data show that in 2016 alone, there were more than 260 million patients with chronic diseases in China, and the expenditure on chronic diseases was about 3.2 trillion yuan. Prior to this, China has carried out trials in 13 provinces and 24 cities (counties) including Shanghai, Jiangsu, Guangdong, Zhejiang and Shandong to guarantee the full expenditure of essential drugs, and provided basic drugs for chronic diseases to residents free of charge as public products. For example, from 2018, medical insurance participants in Jinan, Shandong province, will be able to get five kinds of drugs for treating hypertension, diabetes and coronary heart disease for free. Experts said that the implementation of the policy would help people with chronic diseases take medicines in time and ensure social stability. ${ }^{14}$

In the field of basic drugs, more attention should be paid to the improvement of drug price formation system, especially on preventing drug shortages as well as inhibiting monopoly. The price should also ensure reasonable profits of pharmaceutical enterprises in case of reduced enthusiasm.

\section{Potential Issues}

\section{Selection}

The WHO essential medicine selection criteria are established according to evidence-based medicine. In the selection process, respectively, the variety of the drug use, conveniences, storage, compliance, safety, efficacy and economy will be scored with a special form. Finally, medicines with good grades are included in the list of essential medicines. WHO also has high requirements on stuffs that are responsible for approving the inclusion of the drugs into the list.

At present, the experts hired by our country are generally clinical and pharmaceutical experts of large hospitals in key regions. Due to regional differences and the limitations of the selection of expert group members, the selection of essential drugs has certain limitations, which may not meet the needs of the broad masses nationwide. What's more, experts are not evaluated publicly, thus supervision is hard to be carried out.

The selection of Chinese patent medicines is based on clinical experience, and no large-scale clinical trials have been conducted, which leaves apparent loophole for it.

Most of the drugs in the current NEML are cheap and affordable for patients, but most of the necessary and expensive drugs with good efficacy are not included in NEML.
The top priority in drug selection is to relieve the burden of purchasing these drugs.

\section{Supplement}

Drug production in China is mainly composed of generic drugs, and supply usually exceeds demand. With the medical reform continuously strengthening, the medical market gradually becomes standardized, pharmaceutical production expenditure continues to increase, the market competition is increasing fiercely, and drug prices have dropped. Those factors make some emergency drugs from NEML out of stock.

On the other hand, the emergence of a large number of mature generic drugs with overcapacity technologies has resulted in serious duplication of production and increased market competition.

There are also many problems in the process of drug bidding and procurement. For example, the status of medical institutions is not equal to that of drug manufacturers, and the bid inviters set limitations of price, quality, supply and other restrictions on the bidder, but the bidder is unable to make any restrictions on the bid inviters.

In terms of production, the cost is directly related to the output. If usage decreases, the production cost will inevitably rise, which will greatly reduce the enthusiasm of the bidwinning manufacturer. At the same time of increasing production costs, the phenomenon of lowering prices in the bidding process is common. Unreasonable bidding forms will also directly lead to the low enthusiasm and low profit of drug production enterprises with low price and little use of basic drugs.

In addition, the added social costs generated in the bidding process, such as intermediary agency intervention, local protection, administrative intervention and failure to take care of the particularity of clinical medication, are prominent problems in the bidding of essential drugs.

At the same time, the reform of the medical system will be affected. In terms of drug sales, profit orientation is relatively prominent. The vivosphere of drugs with low profits is getting smaller and smaller, which directly leads to some obstacles in the supply of essential drugs. First, manufacturers are reluctant to produce essential drugs of small varieties and drugs with low incomes, thus the shortage of those drugs has gradually emerged. At the same time, pharmacies are reluctant to deal with essential medicines, which are directly related to their low profits. As the main channel of drug retail in China, hospitals adjust the price of purchased drugs according to a certain proportion and sell them to consumers. Driven by profits, medical institutions are reluctant to purchase basic medicines with lower prices and lower profits. 


\section{Propaganda}

We propose that the Chinese government departments could pay more attention to the implementation of the essential drug system, and expand awareness and implementation of the system.

In terms of medical staff, many front-line clinical workers are not familiar with the national essential drug catalog and its policies. The model of "drug-maintaining-medicine" will also promote doctors to choose drugs with higher prices and avoid basic drugs with small profit and low prices.

For the public, they have no idea what essential drugs are, and have no idea about relevant policies and regulations. The patient has insufficient cognition and is unable to request medication.

\section{Acknowledgments}

This work was supported by the Science and Technology Commission of Shanghai Municipality 2018 YIXUEYINGDAO Project No. 18401902200 (to Ling Wang), the Shanghai Committee of The China Democratic League No. 02054 (to Ling Wang), the National Natural Science Foundation of China No. 31571196 (to Ling Wang), the Science and Technology Commission of Shanghai Municipality 2015 YIXUEYINGDAO Project No. 15401932200 (to Ling Wang), the FY2008 JSPS Postdoctoral Fellowship for Foreign Researchers P08471 (to Ling Wang), the National Natural Science Foundation of China No. 30801502 (to Ling Wang) the Shanghai Pujiang Program No. 11PJ1401900 (to Ling Wang) and Development Project of Shanghai Peak Disciplines-Integrative Medicine No. 20150407.

\section{Conflict of Interest}

We declare that we have no conflict of interest to this work.

\section{References}

1. WHO Expert Committee on the Selection of Essential Drugs. The selection of essential drugs: Report of a WHO expert committee. WHO Technical Report Series. Geneva: WHO, 1977:1-36.
2. Welfare Government of India. National Essential Medicine List (NEML). https://www.pharmacy.gov.my/v2/en/documents/national-essential-medicine-list-neml.html. Accessed on 6 September 2016.

3. Laing R, Waning B, Gray A, Ford N, Hoen ET. 25 years of the WHO essential medicines lists: Progress and challenges. Lancet 2003;361:1723-9.

4. Tang Y, Liu C, Zhang X. Delivery of essential medicines to primary care institutions and its association with procurement volume and price: A case study in Hubei Province, China. Appl Health Econ Health Policy 2017;15:57-64.

5. Yu SY, Shang FF. Comparison between the national essential drugs directory and the drug directory of basic medical insurance. J Health Econ Res 2016;54-6. (in Chinese).

6. Bourliere M, Gordon SC, Flamm SL, et al. Sofosbuvir, Velpatasvir, and Voxilaprevir for previously treated HCV infection. N Engl J Med 2017;376:2134-46.

7. Yu AF, Manrique C, Pun S, et al. Cardiac safety of paclitaxel plus trastuzumab and pertuzumab in patients with HER2positive metastatic breast cancer. Oncologist 2016;21:418-24.

8. Smyth LM, Iyengar NM, Chen MF, et al. Weekly paclitaxel with trastuzumab and pertuzumab in patients with HER2overexpressing metastatic breast cancer: Overall survival and updated progression-free survival results from a phase II study. Breast Cancer Res Treat 2016;158:91-7.

9. Ma L. HER2 testing, clinical pathological analysis and epidemiologic study in Chinese breast cancer patients. China: Peking Union Medical College, 2012. (in Chinese).

10. Tang XL, Zhong L. Tumor-targeting drugs are no longer out of reach. J Health News 2017;008:1. (in Chinese).

11. Yao JJ. The new base drug catalogue clearly supports the development of traditional Chinese medicine, and granule formula will be a strong assist. http://www.zyzhan.com/news/ detail/72667.html. Accessed on 3 December 2018 (in Chinese).

12. Saxena V, Koraishy FM, Sise ME, et al. Safety and efficacy of sofosbuvir-containing regimens in hepatitis C-infected patients with impaired renal function. Liver Int 2016;36:807-16.

13. The central people's government of the People's Republic of China. Premier li keqiang chaired an executive meeting of the state council to hear reports on the implementation of tax cuts and fee reduction policies and measures, and decided to push forward new measures to support the development of the real economy. http://www.gov.cn/premier/2018-08/30/content_5317832.html. Accessed on 30 August 2018 (in Chinese).

14. Liu X. Meet the needs of medication, and Respond to lower fees as expected. http://paper.people.com.cn/rmrbhwb/html/ 2018-09/04/content_1879133.html. Accessed on 34 September 2018 (in Chinese). 